\title{
Selecting landmarks in novel environments
}

\author{
Jared Miller • Laura Carlson
}

Published online: 17 November 2010

(C) Psychonomic Society, Inc. 2010

\begin{abstract}
People use salient landmarks when learning a route through a novel environment. However, it is not clear what makes a given landmark salient. In two experiments, subjects learned a route through a virtual museum, performed a recognition memory test for objects in the museum, and provided spatial descriptions and drew maps of the learned route. Objects with strong perceptual features occurred at decision points or at non-decision points along the route. Objects with both of these features were recognized faster and were included more often in the maps and written directions. When these features were separated, perceptual features maintained a strong influence on the recognition task, but had no influence on the spatial tasks, which were influenced only by spatial features. These findings challenge the idea that either a recognition task or descriptive task alone provides a complete account of landmark representation.
\end{abstract}

Keywords Landmarks · Salience $\cdot$ Spatial features $\cdot$

Navigation $\cdot$ Route $\cdot$ Perceptual features $\cdot$ Spatial language

Landmarks support a variety of cognitive tasks including spatial memory, giving directions, and navigation (Presson \& Montello, 1988; Siegel \& White, 1975). For example, landmarks are used as directional cues that enable navigators to track their spatial position along a route (Chown, Kaplan,

We thank Claire Connell, Kevin Kimberly, Maria Luna, Kevin Mickey, Tiffany Moy, Corinne Swearingen and Rick Ward for their assistance in data collection, and Mike Villano for assistance in developing the virtual environment.

J. Miller $(\bowtie) \cdot$ L. Carlson

Department of Psychology, University of Notre Dame,

Notre Dame, IN 46556, USA

e-mail: jmille39@nd.edu
\& Kortenkamp, 1995; Montello, 1998). In this article, we ask what features of these objects lead to their selection as landmarks, focusing on spatial and perceptual features. ${ }^{1}$

\section{Spatial and perceptual features}

For spatial features, objects at decision points (locations along the route where a decision needs to be made, such as an intersection) are more likely to be included in a description of the environment than objects at non-decision points (Blades \& Medlicott, 1992; Cohen \& Schuepfer, 1980; Denis, Pazzaglia, Cornoldi, \& Bertolo, 1999). Decision point landmarks are also rated as more important to route learning (Daniel \& Denis, 1998) and are recognized more quickly in a recognition test (Janzen, 2006). Such benefits have been attributed to navigational relevance.

For perceptual features, color and size may also influence landmark selection (Allen, Siegel, \& Rosinski, 1978; Blocher \& Stopp, 1998; Talmy, 1983). For example, the more visually conspicuous an object is relative to its environment, the more likely it is to be used as a landmark (Sorrows \& Hirtle, 1999). Furthermore, Nothegger, Winter \& Raubal's (2004) model of landmark selection includes measures of salience defined by form and color.

\section{Previous methodological approaches}

Most previous research examining landmark selection has adopted a methodology in which subjects learn a route

\footnotetext{
${ }^{1}$ The design included a between-subjects manipulation of instructions to focus on the objects, the route, or both during learning; however, we collapsed across this variable because it did not influence the results.
} 
through an environment and provide a description or map of that route (Cohen \& Schuepfer, 1980; Lee, Tappe, \& Klippel, 2002; Raubal \& Winter, 2002), or take a speeded recognition test for objects in the environment (Janzen, 2006; Janzen, Jansen, \& van Turennout, 2008). There are potential limitations to each approach when used separately. For example, a spatial description may under-represent the information learned about the route, because it would be adaptive when giving directions to include only a subset of objects. This is a methodological limitation, because one needs to know what information is represented to best understand how particular landmarks are selected. For example, the inclusion of a given object in a description may reflect the fact that it was the only object represented from that part of the route, or that it was the best of several objects represented. Without knowing whether other objects were represented, and how the selected object differed from those objects, one cannot define the features leading to selection. Conversely, when participants perform a recognition test, one has the best sense of what information has been encoded objects that are correctly recognized. However, one still needs a means for inferring the basis on which objects from this set are selected as landmarks. Some objects may be recognized better than others, but this may be due to features that are not important for navigation or landmark selection.

In the current study, we combine both of these approaches, using a recognition measure to identify objects that are encoded and considered privileged (responded to faster and more accurately) and then collecting spatial descriptions and map drawings to assess whether the privileged objects are more likely to be included as landmarks. According to the framework by Caduff and Timpf (2008), if different features are prioritized across these tasks, there will be no correspondence in performance in terms of the relative importance of the features.

Finally, previous work (Nothegger, Winter, \& Raubal, 2004; Raubal \& Winter, 2002) has examined the role of spatial and perceptual features on landmark selection in natural environments, where such features are difficult to control, or in virtual environments where perceptual features were neutralized and thus not pitted against spatial features (Janzen, 2006), leading to independent examinations of the roles of spatial and perceptual features in the absence of competition. To better assess their relative importance for landmark selection, we examined conditions in which these features were in conflict (Experiment 1) or in concert (Experiment 2).

Participants learned a route through a virtual museum in which they passed by tables containing objects (see Fig. 1, panel a for a first person view), performed a speeded recognition test for these objects, and provided descriptions and maps of the route. Objects at non-decision points (NDP) were significantly more perceptually salient (see below) than objects at decision points that occurred at intersections either

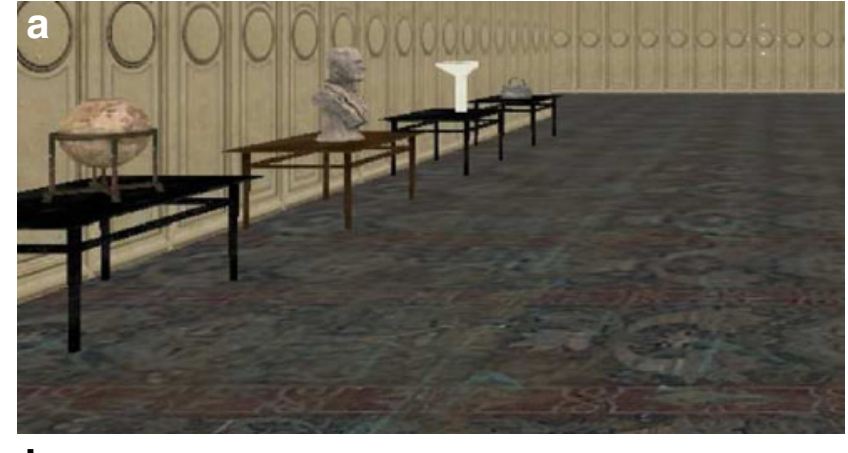

b

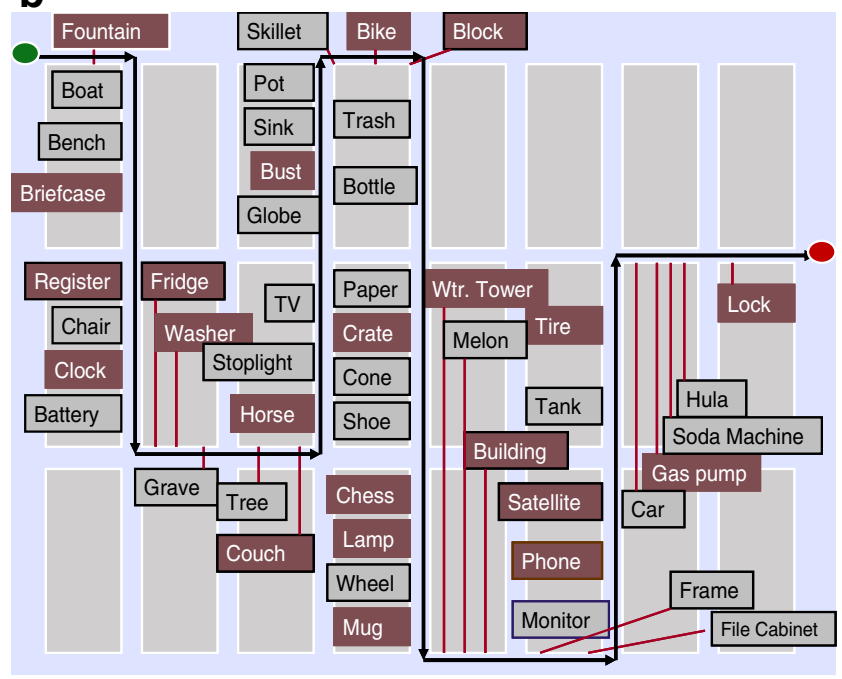

Fig. 1 Panel $\boldsymbol{a}$ shows a first-person view of one corridor of the museum, with a globe, bust, sink, and teakettle on the four tables. Panel $\boldsymbol{b}$ shows an overhead schematic view of the structure of the museum. The green dot shows the starting point of the route; the black line shows the path, and the red dot shows the ending point. The larger rectangles are tables that line the corridors of the museum, with the object labels identifying the objects (labels were not presented to participants). Brown boxes on top of the tables represent the objects placed on brown tables, and gray boxes represent objects placed on black tables

requiring a turn (DP-turn) or requiring no turn (DP-noturn). In Fig. 1, panel b, the battery is a DP-turn object, the chair is a DP-noturn object, and the cash register is a NDP object. This competition between spatial and perceptual features enables an assessment of the relative importance of these features for recognition and for spatial descriptions and map drawings. Following Janzen (2006), participants were told to learn a subset of objects (in our paradigm, objects on either brown or black tables) to ensure that the objects in each condition were equally attended.

\section{Method}

Participants Ninety-six undergraduates from the University of Notre Dame participated. All gave informed consent and were treated in accordance with APA ethical guidelines. 
Stimuli The environment was a virtual museum consisting of 13 hallways with 16 intersections, created using the software "Half Life 2" (see Fig. 1). Turns were made at eight intersections. Fifty tables (half brown and half black) were placed in the hallways against the walls, with one object placed on each table. Each hallway contained two, three, or four objects. Additional tables with dummy objects occurred around walls and down hallways that were visible from the path. There were a total of 48 critical objects (16 DP-turn, 16 DP-noturn, and 16 NDP). A first person movie navigating the route was created, with a frame rate of 30 frames/s, presented on a $19-\mathrm{inch}, 60-\mathrm{Hz}$, color monitor. Mean viewing time for each object was $7.6 \mathrm{~s}$, with no difference across navigational relevance (DP-turn, $M=7.65$; DP-noturn, $M=7.58$; NDP, $M=7.57$ ).

For perceptual salience, in designing the stimuli, we defined perceptual salience largely on the basis of size and color. Specifically, the salient objects were $68 \%$ larger than their surrounding objects, based on a comparison of the areas computed by a bounding box enclosing each object. In addition, the salient objects were uniquely colored with respect to the surrounding objects or background in 12 of the 17 corridors. To verify our designations of salience and to assess how salience varied across navigational relevance, an independent group of subjects $(N=50)$ provided perceptual ranks for each object. These subjects viewed head-on images of each wall in the museum (see Fig. 2) and rank ordered each object based on "how much it popped out relative to the other objects." There were six walls that contained two objects, six walls that contained three objects, and five walls that contained four objects. Ranks were standardized by subjects to account for the different number of objects in a hallway; for two objects, the values were 1 and 0 for the high and low ranked objects; for three objects, the values were $1, .5$, and 0 ; and for four objects, the values were $1, .66, .33$, and 0 . The perceptual salience rank for NDP objects $(M=.71, \mathrm{SE}=.05)$ was significantly greater than for both DP-turn $(M=.40$, $\mathrm{SE}=.06)$ and DP-noturn $(M=.39, \mathrm{SE}=.05)$ objects, $p<$ .01 . Thus, higher perceptual salience was associated with lower navigational relevance, establishing competition between these features.

Procedure Participants were told that they were guides for a museum, and that they were going to lead a tour that highlighted the objects on the brown tables or black tables. They were asked to learn the route through the museum and memorize the identities and locations of the objects on the brown/black tables. Before learning the route, subjects viewed a slide show with each object in the museum shown in isolation with a label. This was done in order to insure that each object was easily recognizable and identifiable.
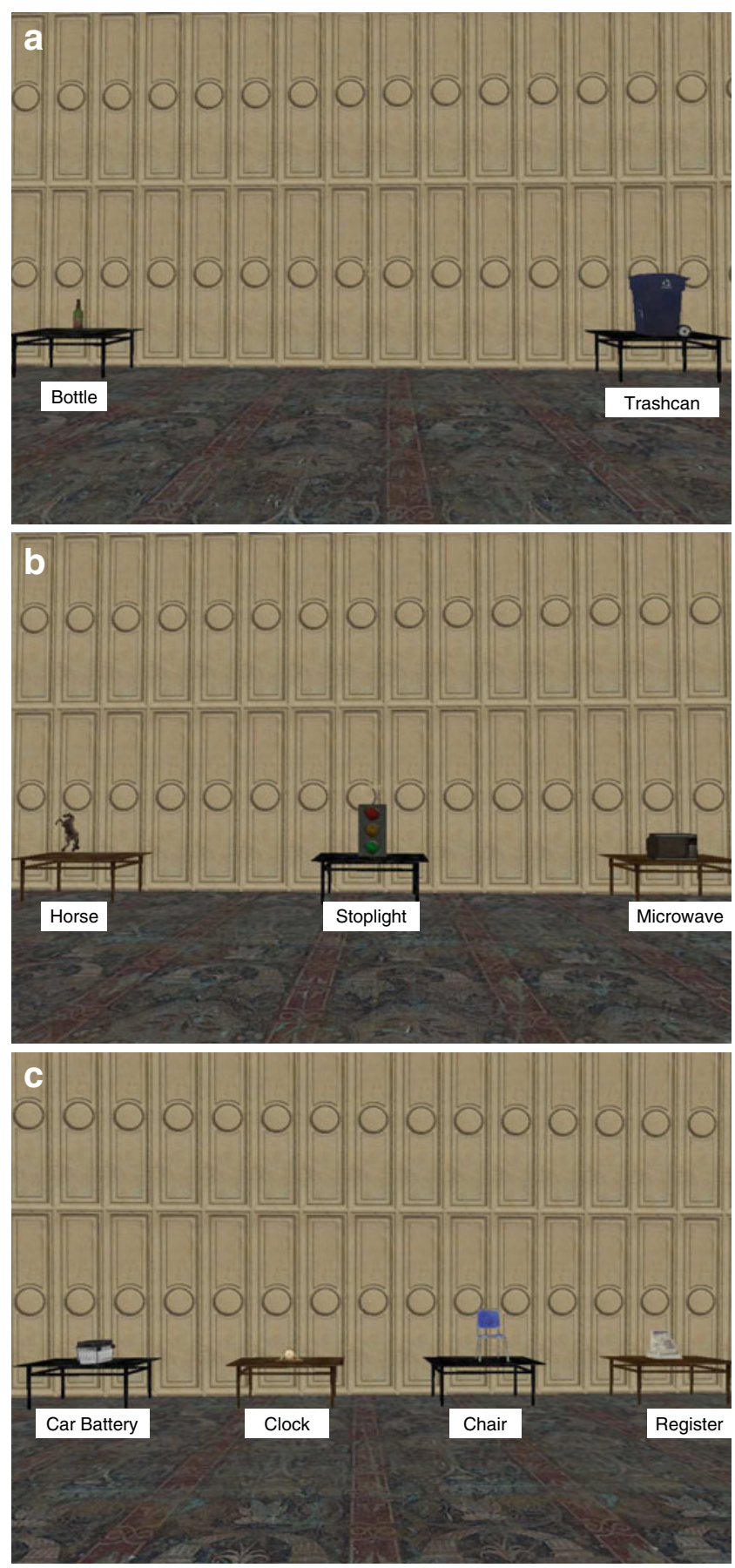

Fig. 2 Sample hallways showing objects used during the perceptual rank test. Labels of the objects are included for clarity; subjects viewed the pictures with the object labels on a separate sheet. Panel $\boldsymbol{a}$ : A two-object hallway; subjects ranked the trashcan as more perceptually salient than the bottle. Panel $\boldsymbol{b}$ : A three-object hallway; the stoplight was ranked as the most perceptually salient object. Panel $c$ : A four-object hallway; the chair was ranked as the most perceptually salient object

The slide show also included the objects that were not viewed in the museum but served as distractors on the recognition test. Subjects then viewed a first-person movie of the route five times and were told to attend to the objects 
on their designated tables. After viewing the movie, subjects performed a recognition task consisting of 82 trials (48 critical objects and 34 filler objects). Each trial consisted of a presentation of a fixation cross for $100 \mathrm{~ms}$, followed by a blank white screen for $250 \mathrm{~ms}$, and then the object in a canonical view on a white background. Subjects responded as quickly as possible to the query "Was this object in the museum?" indicating a 'yes' or 'no' responses by pressing handheld switches. Assignment of switches to hand was counterbalanced across subjects. Finally, participants gave written directions of the tour through the museum to an imaginary coworker and drew a map, with the instruction to mention all objects that were remembered. The order of the spatial description and map task was counterbalanced across subjects.

\section{Results and discussion}

To combine data across tour type, objects on black and brown tables were reclassified as "attended" or "nonattended" (e.g., for the black table tour, the black table objects were "attended" and brown table objects were "nonattended"). For the recognition task, data were excluded from the response time analysis if response times exceeded three standard deviations from the mean $(<1 \%$ for both Experiments 1 and 2).

Recognition task Subjects were faster and more accurate for objects placed on attended tables $(M=791 \mathrm{~ms}, S E=$ $10.1 ; M=83.1 \%, S E=1.40)$ than non-attended tables $(M=$ $861 \mathrm{~ms}, S E=12.3 ; M=48.22 \%, S E=1.70), F(1,95)=$ 26.7, $p<.01$ and $F(1,95)=111.1, p<.001$. Because accuracy for the non-attended tables did not depart from chance $(50 \%, p>.05)$, we focused on the attended tables. $^{2}$

Mean correct "yes" responses are shown in Fig. 3. There was no significant effect of navigational relevance, either in accuracy (Fig. 3a), $F(2,190)=2.2, p>.05$ or in response times (Fig. 3b) $F(2,190)=.47, p>.05$. With the NDP objects more perceptually salient than DP objects, we failed to replicate Janzen's (2006) privileged recognition for DP objects. In addition, the perceptual ranks for correctly recognized objects $(M=.52, S E=.005)$ were higher than the perceptual ranks for objects that participants failed to recognize $(M=.44, S E=.006), F(1,95)=88.7, p<.05)$, with this effect occurring within each navigational relevant category, all $p s<.05$ (see top row, Table 1). This suggests that perceptual features were encoded during learning and

\footnotetext{
${ }^{2}$ Note that results including the non-attended tables show the same patterns in this and all other analyses.
}

played a significant role in recognition, regardless of navigational relevance.

Spatial tasks Subjects included more attended objects in their spatial descriptions $(M=3.66, S E=.15)$ and maps $(M=3.94, S E=.15)$ than non-attended objects $(M=.75$, $S E=.07 ; M=.85, S E=.08), F(1,95)=114.7, p<.05$; $F(1,95)=124.2, p<.05$, respectively. For consistency with the recognition task, we focus on the attended objects.

The means for included objects are shown in Fig. 3. There was a main effect of navigational relevance in both the spatial description task (Fig. 3c), $F(2,190)=5.91, p<$ .05 , and the map drawing task (Fig. 3d), $F(2,190)=3.41$, $p<.05$, with planned comparisons revealing that DP-turn objects were included more frequently than NDP objects (spatial description task: DP-turn, $M=3.97, S E=.26$ vs. NDP, $M=3.38, S E=.27 ; p<.05$ and map drawing task: DP-turn, $M=4.21, S E=.25$ vs. NDP, $M=3.71, S E=$ .27). Thus, there was a bias to include decision-point objects in these spatial tasks. In contrast, there was no significant effect of perceptual salience (spatial description task: included, $M=.51, \mathrm{SE}=.009$ versus omitted, $M=$ $.50, S E=.003 ; F(1,95)=.240, p>.05 ;$ map drawing task: included, $M=.51, S E=.008$ versus omitted, $.50, S E=$ $.003 ; F(1,95)=.716, p>.05)$. Furthermore, as shown in Table 1, there was no effect of perceptual salience within any level of navigational relevance in either task, $p s>.05$. These data suggest that perceptual features did not influence the spatial tasks, despite the fact that these features were encoded during learning and significantly influenced recognition.

\section{Predictive ability of the recognition task}

The findings that perceptual salience influences the recognition task, while having no influence on the spatial description tasks, and conversely that navigational relevance influences the spatial tasks but not the recognition task, are troublesome for predictions regarding landmark selection that are generated in one task and applied to the other task. Indeed, an items analysis showed no relation between the reaction time for objects in the recognition task and the likelihood that the object was included in either the spatial description task, $F(1,46)=1.45, p>.05$, or the mapping drawing task, $F(1,46)=1.23, p>.05$.

One question raised by Experiment 1 is whether the differential influence of the features across the two types of tasks is an artifact of the competition that we created. To address this, we switched the locations of objects in the museum to equate perceptual salience for the DP-turn and NDP objects. Nevertheless, because there is a range of 
Fig. 3 Data for Experiment 1, plotted as a function of navigational relevance. Panel a: Mean percent of objects correctly recognized. Panel $\boldsymbol{b}$ : Recognition time (in ms) for correctly recognized objects. Panel $c$ : Mean number of objects included in the spatial description task. Panel $\boldsymbol{d}$ : Mean number of objects included in the map drawing tasks
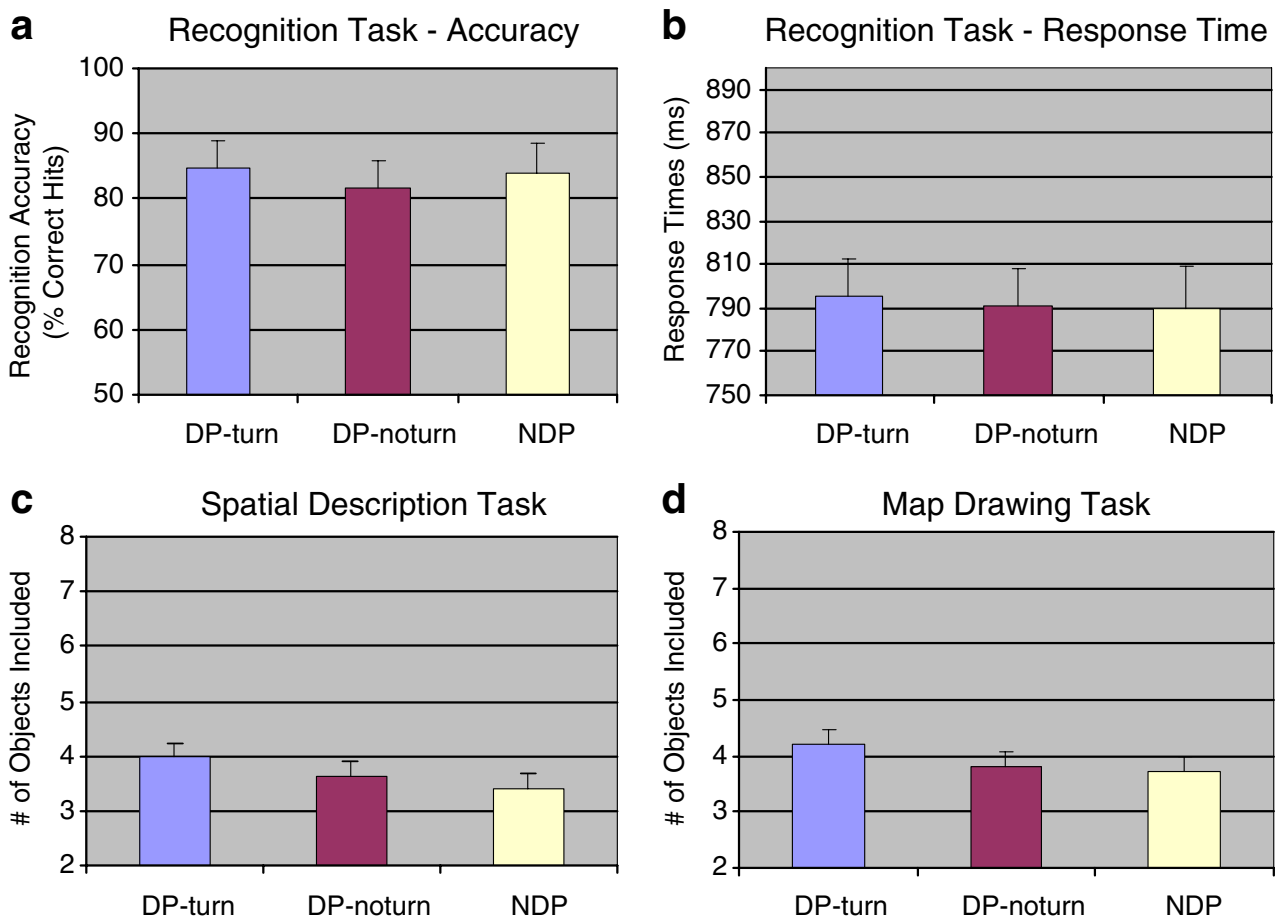

perceptual ranks within each navigationally relevant category, we can look within each category (thus holding navigational relevance constant) and assess any influence of perceptual features across the tasks.

Table 1 Standardized perceptual salience rank of objects correctly recognized or included as a function of task and navigational relevance

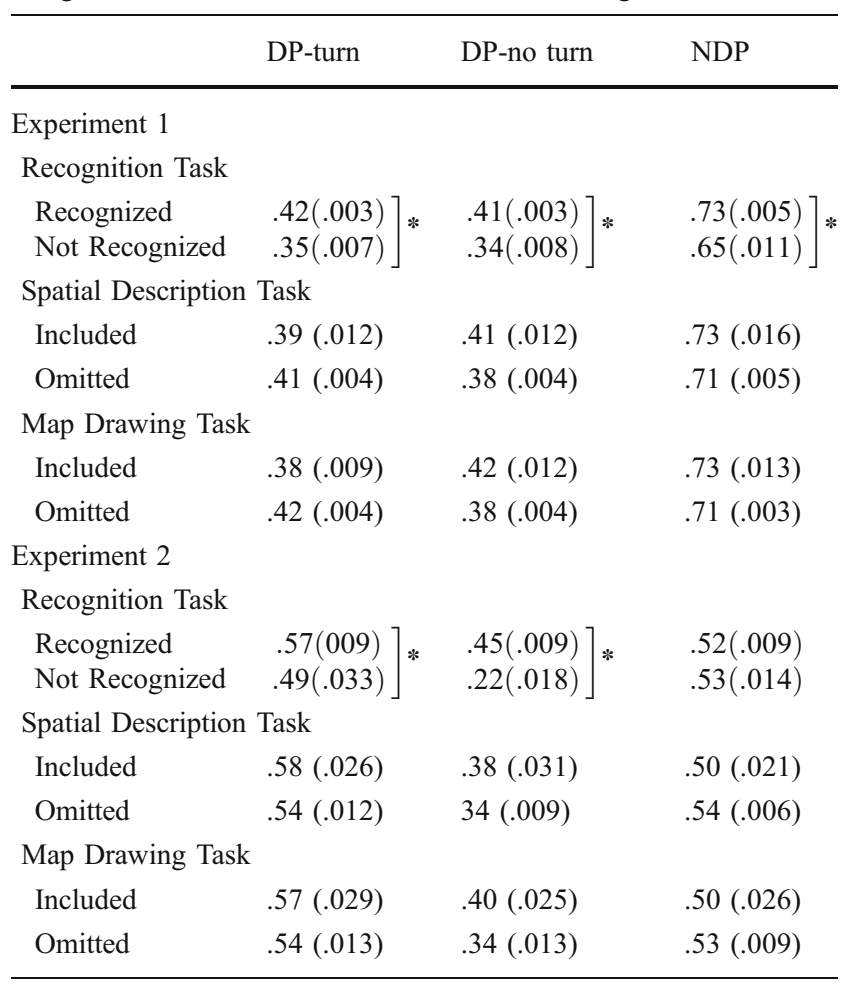

\section{Method}

Subjects

Thirty-two undergraduates from the University of Notre Dame participated. All gave informed consent and were treated in accordance with APA ethical guidelines.

Apparatus, stimuli, and procedure

All details were as with Experiment 1 except that the positions of objects on tables were altered to equate navigational relevance. This was verified by no difference in perceptual salience ranks [DP-turn objects $(M=.55, \mathrm{SE}=.09)$; NDP $(M=.53, \mathrm{SE}=.07), p>.05]$ provided by a new set of naïve subjects $(N=50)$.

\section{Results and discussion}

Recognition task Subjects were faster and more accurate for objects placed on attended tables $(M=812 \mathrm{~ms}, S E=$ $16.5 ; M=82.9 \%, S E=2.92)$ than non-attended tables $(M=$ $863, S E=18.4 ; M=46.4 \%, S E=4.15), F(1,31)=4.83, p<$ $.05, F(1,31)=59.2, p<.05$. We focus on the attended tables, because accuracy for the non-attended tables did not depart from chance $(50 \%, p>.05)$.

Mean correct "yes" responses are shown in Fig. 4. There was no significant effect of navigational relevance on accuracy (Fig. 4a), $F(2,62)=1.5, p=.231$, but consistent 
Fig. 4 Data for Experiment 2, plotted as a function of navigational relevance. Panel a: Mean percent of objects correctly recognized. Panel $\boldsymbol{b}$ : Recognition time (in ms) for correctly recognized objects. Panel $\boldsymbol{c}$ : Mean number of objects included in the spatial description task. Panel d: Mean number of objects included in the map drawing tasks
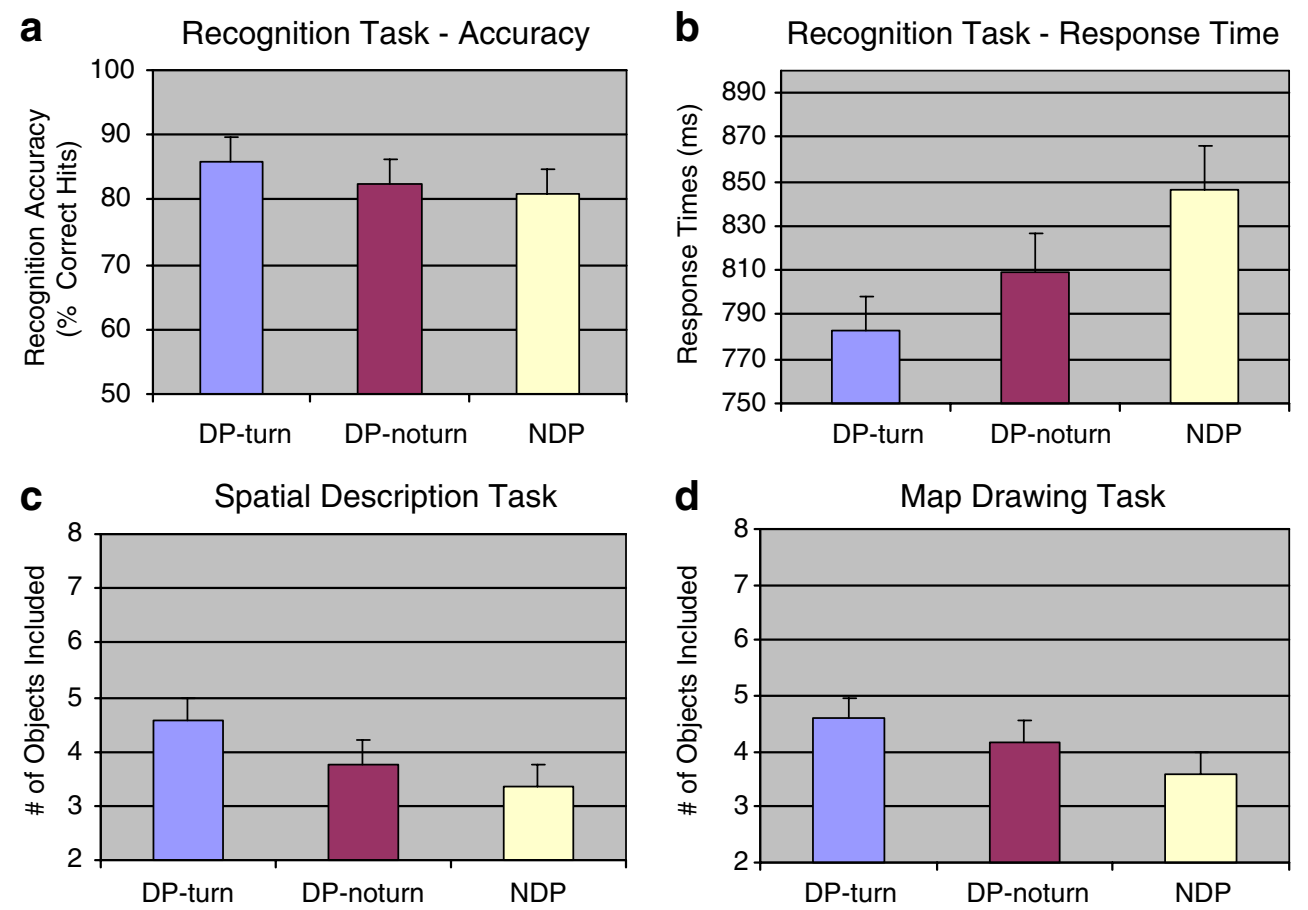

with Janzen (2006) there was an effect on response time (Fig. 4b), $F(2,62)=3.24, p<.05$, with recognition of DPturn objects $(M=783 \mathrm{~ms}, S E=21.1)$ significantly faster than NDP objects $(M=846, S E=19.9), p<.05$. Additionally, there was a significant effect of perceptual salience, with the perceptual ranks for correctly recognized objects $(M=.52, \mathrm{SE}=.005)$ higher than the perceptual ranks for objects that were not recognized $(M=.42, \mathrm{SE}=$ $.015), F(1,31)=26.7, p<.05$. Furthermore, this effect held within DP-turn and DP-noturn categories, $p<.05$ (see Table 1). These results are consistent with Experiment 1, suggesting that perceptual features were encoded during learning and played a significant role in the recognition task.

Spatial tasks Subjects included more attended objects $(M=$ 3.91, $S E=.26)$ in their spatial descriptions and maps $(M=$ 4.11, $S E=.23)$ than non-attended objects $(M=.96, S E=.15$; $M=1.18, S E=.15), F(1,31)=40.3, p<.05 ; F(1,31)=47.1$, $p<.05$, respectively. As in Experiment 1, and for consistency with the recognition task, we focus on the attended objects.

The means for included objects are shown in Fig. 4. There was a main effect of navigational relevance in both the spatial description task (Fig. 4c), $F(2,62)=8.68, p<.05$, and the map drawing task (Fig. $4 \mathrm{~d}$ ), $F(2,62)=5.71, p<.05$, with DP-turn objects included more frequently than NDP objects (spatial description: $M=4.59, S E=.39$ and $M=$ 3.34, $S E=.43$, respectively, $p<.05$; map drawing task: $M=$ 4.59, $S E=.36$ and $M=3.59, S E=.41$, respectively, $p<$ $.05)$. In contrast, there was no significant effect of perceptual salience on objects that were included versus omitted, in either the spatial description task: included, $M=.49, \mathrm{SE}=$
.014 versus omitted, $M=.47, \mathrm{SE}=.006 ; F(1,31)=.830, p>$ .05 ; or the map drawing task: included, $M=.49, \mathrm{SE}=.012$ versus omitted, $M=.47, \mathrm{SE}=.007 ; F(1,31)=1.61, p>.05$. Furthermore, there was no effect of perceptual salience within any level of navigational relevance, $p s>.05$ (see Table 1). This is consistent with Experiment 1 and further suggests that perceptual features did not influence the spatial tasks.

\section{Predictive ability of the recognition task}

In an items analysis, recognition time was predictive of whether that object was included in the spatial description task, $F(1,46)=14.27, p<.05$, and the mapping task, $F(1,46)=9.02, p<.05$, accounting for $22.6 \%$ and $14.6 \%$ of the variance, respectively. This is likely due to navigational relevance. Nevertheless, perceptual features continued to influence which objects were likely to be remembered within navigational relevance, but had no influence on the spatial tasks. This suggests that the two tasks led to very different conclusions about the prioritization of features for the selection of landmarks. Perceptual features may influence whether a landmark is encoded, but spatial features influence whether it is selected during spatial tasks.

\section{General discussion}

The first goal of this work was to assess what features of objects made them more likely to be selected as landmarks 
during route learning. Experiments 1 and 2 demonstrated that the influences of spatial and perceptual features varied as a function of task. Navigational relevance affected all tasks in Experiment 2, whereas perceptual salience only affected the recognition task. Objects that were judged more perceptually salient were more likely to be correctly recognized, but this did not impact whether they were included in the spatial tasks. Furthermore, the effect of perceptual features in Experiment 1 was strong enough to eliminate the effect of navigational relevance demonstrated by Janzen (2006) when these were in competition.

The finding that spatial features robustly affected landmark inclusion in spatial descriptions of environments is consistent with previous work (Blades \& Medlicott, 1992; Denis et al., 1999; Raubal \& Winter, 2002). Furthermore, the finding that perceptual features can affect landmark recognition is consistent with theories and models that argue perceptual salience is a critical dimension of landmarks (Nothegger, Winter, \& Raubal, 2005; Raubal \& Winter, 2002; Sorrows \& Hirtle, 1999). The most interesting and important contribution of these experiments is the dissociation between which objects are assumed to be privileged in the representation that one builds during route learning (as measured by the recognition task) and which objects are assumed to be privileged as landmarks when using this representation to provide descriptions or draw maps.

This dissociation can be discussed within in Caduff and Timpf's (2008) descriptive framework of landmark salience. They define salience using a three-component Saliency Vector consisting of perceptual, cognitive, and contextual salience. Perceptual salience refers to the potential of an object to attract attention based on its environmental properties (e.g., size, color, shape, spatial context). Cognitive salience refers to the directing of attention in an endogenous manner based on knowledge. These two types of salience influence the objects that are encoded during navigation and the objects that are retrieved, weighted by a degree of recognition (how identifiable the object is) and its idiosyncratic relevance (the object's personal or historical significance to the observer). Contextual salience refers to how context affects the allocation of attention to particular landmarks, with one aspect related to task demands. Task-based context operates to optimize the choice of a landmark for a given task, and this can influence are all other components of the Saliency Vector.

As applied to the current experiments, when navigation is important to the task, spatial features will likely have a strong influence on landmark selection. This is consistent with our finding that navigational relevance influences the spatial tasks in Experiments 1 and 2. Similarly, when the task prioritizes perceptual features (as necessary for recognition), these features should have a strong influence, as in Experiments 1 and 2 with demonstrated effects of perceptual features on landmark recognition.
The second goal of the current study was to determine whether a recognition task could be used to infer which objects are likely to be included as landmarks in spatial tasks. Experiment 1 found no correlation between the objects privileged in the recognition and objects included in the spatial tasks. Furthermore, perceptual features had a differential influence on the two tasks. Although Experiment 2 found a significant correlation between the two tasks (perhaps not surprising because perceptual features were controlled), there was still an influence of perceptual features on the recognition task but not the spatial tasks. These experiments thus provide initial empirical support for Caduff and Timpf's (2008) suggestion of contextual salience. The salience of an object and its likelihood to be selected as a landmark depend not simply on it features relative to other objects in the environment (perceptual salience), but also critically on the mapping of these features onto the demands of the task (contextual salience).

\section{References}

Allen, G. L., Siegel, A. W., \& Rosinski, R. R. (1978). The role of perceptual context in structuring spatial knowledge. Journal of Experimental Psychology: Human Learning and Memory, 4, 617-630. doi:10.1037//0278-7393.4.6.617

Blades, M., \& Medlicott, L. (1992). Developmental differences in the ability to give route directions from a map. Journal of Environmental Psychology, 12, 175-185. doi:10.1016/S0272-4944(05)80069-6

Blocher, A., \& Stopp, E. (1998). Time-dependent generation of minimal sets of spatial descriptions. In P. Olivier \& K.-P. Gapp (Eds.), Representation and processing of spatial relations (pp. 57-72). Mahwah, NJ: Erlbaum.

Caduff, D., \& Timpf, S. (2008). On the assessment of landmark salience for human navigation. Cognitive Processing, 9, 249267. doi:10.1007/s10339-007-0199-2

Chown, E., Kaplan, S., \& Kortenkamp, D. (1995). Prototypes, Location and Associative Networks (PLAN): Towards a unified theory of cognitive mapping. The Journal of Cognitive Science, 19, 1-51. doi:10.1016/0364-0213(95)90003-9

Cohen, R., \& Schuepfer, T. (1980). The representation of landmarks and routes. Child Development, 51, 1065-1071. doi:10.2307/1129545

Daniel, M. P., \& Denis, M. (1998). Spatial descriptions as navigational aids: A cognitive analysis of route directions. Cognitive Science, 7, 45-52. doi:10.1007/s001970050050

Denis, M., Pazzaglia, F., Cornoldi, C., \& Bertolo, L. (1999). Spatial discourse and navigation: An analysis of route directions in the city of Venice. Applied Cognitive Science, 13, 145-174. doi:10.1002/ (SICI)1099-0720(199904)13:2<145::AID-ACP550>3.0.CO;2-4

Janzen, G. (2006). Memory for object location and route direction in virtual large scale space. The Quarterly Journal of Experimental Psychology, 59, 493-508. doi:10.1080/02724980443000746

Janzen, G., Jansen, C., \& van Turennout, M. (2008). Memory consolidation of landmarks in good navigators. Hippocampus, 18, 40-47. doi:10.1002/hipo.20364

Lee, P. U., Tappe, H., \& Klippel, A. (2002). Acquisition of landmark knowledge from static and dynamic presentation of route maps. In: W. Gray \& C. Schunn (Eds.), Twenty-fourth annual conference of the cognitive science society (pp. 32-34).

Montello, D. R. (1998). A new framework for understanding the acquisition of spatial knowledge in large-scale environments. In M. J. Egenhofer \& R. G. Golledge (Eds.), Spatial and temporal 
reasoning in geographic information systems (pp. 143-154). New York: Oxford University Press.

Nothegger, C., Winter, S., \& Raubal, M. (2004). Selection of salient features for route directions. Spatial Cognition and Computation, 4, 113-136. doi:10.1207/s15427633scc0402 1

Presson, C. C., \& Montello, D. R. (1988). Points of reference in spatial cognition: Stalking the elusive landmark. British Journal of Developmental Psychology, 6, 378-381.

Raubal, M., \& Winter, S. (2002). Enriching wayfinding instructions with local landmarks. In M. J. Egenhofer \& D. M. Mark (Eds.), Geographic information science (pp. 243-259). Berlin, Germany: Springer. doi:10.1007/3-540-45799-2_17
Siegel, A. W., \& White, S. H. (1975). The development of spatial representations of large-scale environments. In H. W. Reese (Ed.), Advances in child development and behavior (pp. 9-55). New York, NY: Academic.

Sorrows, M. E., \& Hirtle, S. C. (1999). The nature of landmarks for real and electronic spaces. In C. Freksa \& D. M. Mark (Eds.), Spatial information theory, volume 1661 of lecture notes in computer science (pp. 37-50). Berlin, Germany: Springer. doi:10.1007/3-540-48384-5_3

Talmy, L. (1983). How Language structures space. In H. Pick \& L. Acredolo (Eds.), Spatial orientation: Theory, research and application (pp. 225-282). New York: Plenum Press. 